\title{
A Clustering Approach for Characterizing Voter Types: An Application to High-Dimensional Ballot and Survey Data
}

\author{
Shiro Kuriwaki*
}

June 25, 2020

\begin{abstract}
Large-scale ballot and survey data hold the potential to uncover the prevalence of swing voters and strong partisans in the electorate. However, existing approaches either employ exploratory analyses that fail to fully leverage the information available in high-dimensional data, or impose a one-dimensional spatial voting model. I derive a clustering algorithm which better captures the probabilistic way in which theories of political behavior conceptualize the swing voter. Building from the canonical finite mixture model, I tailor the model to vote data, for example by allowing uncontested races. I apply this algorithm to actual ballots in the Florida 2000 election and a multi-state survey in 2018. In Palm Beach County, I find that up to 60 percent of voters were straight ticket voters; in the 2018 survey, even higher. The remaining groups of the electorate were likely to cross the party line and split their ticket, but not monolithically: swing voters were more likely to swing for state and local candidates and popular incumbents.
\end{abstract}

* PhD candidate, Department of Government, Harvard University. URL: https://www.shirokuriwaki.com/. I am indebted for Soichiro Yamauchi for his help in the derivation and implementation of the method. I also thank Yuki Atsusaka, Max Goplerud, David Kimball, Jeff Lewis, Kosuke Imai, and Stephen Ansolabehere for helpful ideas and suggestions. 


\section{Introduction}

Finding and labelling voting blocs are ubiquitous in election analysis. Theories of political behavior, especially those explaining electoral change, cluster voters into interpretable prototypes and assign them labels such as core and periphery, standpatters and floating voters (Campbell 1960; Hill and Kriesi 2001; Key 1966; Smidt 2017). Almost instinctively, political consultants, journalists, and election observers latch on to labels such as the "soccer mom" or the "white working class" to construct narratives about voting behavior, even if the label may not have a uniform definition, or may not be the best statistical predictor (Carroll 1999; Cohn 2019; Carnes and Lupu 2020). In particular, a recurring voting bloc in modern accounts of the US electorate is the "swing voter" - a pivotal (and perhaps dwindling) group of voters who are indifferent between either party to a first approximation and are therefore considered persuadable.

But existing approaches to this grouping exercise are either based exclusively on predefined groupings, or on a series of comparisons between votes in pairs of offices. The former risks not fully leveraging the information contained in the data, and the latter simply becomes intractable with high-dimensional large- $N$ datasets with an exceeding number of possible voting patterns.

In this paper I offer an alternative framework: a clustering algorithm that summarizes complex individual-level voting data to interpretable blocs using a probabilistic model. I focus on the specific case of identifying types of voting patterns that include core (party base) and swing, measuring their prevalence, and characterizing the officespecific voting patterns. I derive and implement a fast algorithm tailored to these data structures common in studies of elections in which a single voter votes on multiple offices for federal, state, and local office, each contested by nominees of a major party with varying backgrounds. I then apply this to two datasets: 300,000 actual ballots 
from Palm Beach County in the 2000 general election, and survey data from ten states from the Cooperative Congressional Election Study in 2018.

This paper enhances our understanding of swing voters - how prevalent they are in the current electorate, how likely they are to cross party lines, and what types of voters swing. It has several specific theoretical, substantive, and methodological contributions. Theoretically, following a long tradition of research (Burden and Kimball 2002; Beck et al. 1992), I infer these latent characteristics from patterns in ticket splitting. But instead of arguing deterministically that only ticket splitters are swing voters, I provide a probabilistic definition of the swing voter bloc. Further, these past studies of ticket splitting analyze one pair of offices at a time. I incorporate information from the joint set of votes on multiple offices in identifying the clusters.

Using voting data from a range of offices and elections, I generally find that a clear majority of the electorate can be classified into the partisan base, but a sizable bloc are what we would reasonably label as swing. The size and patterns of this bloc vary systematically. In Palm Beach County, swing voters were more likely to split their ticket in downballot offices rather than high-information Congressional races. In survey data from ten states, I find that up to 8 in 10 of the midterm electorate in 2018 were straight party voters, but popular Governors and US Senators appear to create swing blocs of their own by drawing support from out-partisans.

Methodologically, I show how and when model-based clustering is a powerful tool for empirical discovery and calibration of theories and narratives in studies of political behavior. Despite the natural connections between the parameters in a discrete choice clustering model and the structure of the US ballot, little work has applied this model to voting data, ${ }^{1}$ perhaps due to concerns about interpretability and lack of substantive

1 An exception is a working paper by Dubin and Gerber (1992), who analyze ballot propositions, and Hill and Kriesi (2001) who apply the finite mixture model to longitudinal public opinion data to test Converse's black-and-white model. 
theory. My proposed approach has three methodological features on this point. First, by using a clustering algorithm as opposed to the more standard regression approach, I can properly leverage the information that is contained by the same voter making repeated vote choice decisions between Republicans, Democrats, and abstention (for example) in multiple offices. Second, it embraces the principle of unsupervised learning more so than ideal point models. This entails targeting the parameters of a simple model that best fit the data, instead of modeling the behavior of known or presumed voting blocs. Third, my statistical approach follows a model of political behavior (Ahlquist and Breunig 2012), instead of simply grouping observations that are close on a particular distance metric (Müllner 2013). Analysts still pick the number of clusters to estimate, and must use substantive prior knowledge to guide the interpretation of each cluster. In summary, the main virtue of the clustering approach is that it is a principled framework to leverage the information in high dimensional voting data.

In the remainder of this paper, I describe the clustering approach and what it can reveal about swing voters in the American Politics. In the models and methods section, I set up the model and show how I derive an EM algorithm to measure the parameters in an open-source program, clusterCVR. In the process, I highlight the assumptions and implications of this statistical approach. Then, in both of my applications, I estimate the same parameters and use a visualization that highlights the estimated parameters in a more interpretable fashion. In particular, I find in my two applications that swing voters form a clear voter type, even though the office-specific patterns of how they swing varies by the type of office and experience of the candidate.

\section{Models and Methods}

Two quantities of interest are key in analyzing blocs of core vs. swing. First, what is the proportion of each bloc in the electorate? If the swing voter bloc is too small, 
they may not be pivotal and thus of less interest for campaigns (Grimmer and Marble 2019). Second, what are the latent voting patterns of each cluster? In other words, do most swing voters split their ticket for all downballot races, or do their votes change depending on the particular candidate running in each office?

Clustering analysis — and model-based clustering of categorical outcomes in particular — is a well suited to estimate these quantities of interest guided by a simple model of vote choice. ${ }^{2}$ Political scientists have employed clustering on various types of high-dimensional data such as political institutions, text data, and treatment effects (Estevez-Abe et al. 2010; Grimmer and King 2011; Shiraito 2016; Sewell et al. 2016; for a review see Ahlquist and Breunig 2012) to divide them into a few meaningful prototypes. Clustering is even more widely used in fields such as psychology and marketing (Fiske et al. 2002; Wedel and Kamakura 2000). But they are still less common than regression based methods in political science, so I start with the logic of the basic model that I implement in an open-source R package, clusterCVR, and finally respond to commonly recognized limitations of clustering as a method to analyze political behavior.

\subsection{Main Logic of the Clustering Model}

Instead of defining clusters of voters by predictors of vote choice such as race and education, this paper starts with the case in which we only observe the outcome of votes.

Let $\mathbf{Y}$ be the $N \times J$ vote matrix of voters $i \in\{1, \ldots, N\}$ voting in offices $j \in\{1, \ldots, J\}$. Each vote $Y_{i j}$ takes on a discrete, unordered categorical value $\ell \in\{0, \ldots, L\}$. In this

2 I use the term "clustering analysis" interchangeably with other names in the literature, such as finite mixture models and latent classification analysis. The literature also uses variants of the word "categorical" to mean the same thing: multinomial, discrete, qualitative, or polytomous. In the context of this paper, these all refer to the same data structure, i.e. data that is drawn from a fixed number of unordered categories. 
paper, we focus on vote data where we have coded each vote as

$\ell \in\{$ straight ticket, split ticket, third party, undervote $\}$,

by recoding vote choices based on a reference category, such as vote choice at the top of the ticket (as in my ballot application) or partisan identification (as in my survey example). This recoding makes blocs of partisan attachment clearly visible, but for other research questions one could use outcomes \{undervote, Republican, Democrat, third party just as easily. Our data is also high-dimensional, in the sense that for each voter, we observe $J$ choices on different partisan contests. Instead of analyzing each office separately, we wish to leverage information from across offices to infer a voter's latent voting pattern.

Common tools for clustering and dimension reduction such as $k$-means, PCA, or binary classification cannot be used for such vote choice data because they all require continuous or binary outcomes. Imposing an ordinal scale on vote choice data or discretizing it will likely mask interesting patterns and nuances (Goplerud 2019).

Alternatively, one might analyze vote choice data by showing two-way and even three-way tabulations, computing the proportion of voters that exactly matches a particular voting pattern. However, this quickly becomes intractable because the combinatorics of vote choice on the US long ballot. A typical general election ballot in the US can contain a dozen or so offices, with each voter often making one of at least three choices (Republican, Democrat, and abstain) in each. Third party candidates and the reality that in some offices in some districts are not contested by a major party further increase the number of considerations. Focusing on a pair of offices (e.g. the President and US House, as in Burden and Kimball (2002)) effectively discards valuable information, while enumerating each potential voting pattern (Beck et al. 1992) reduces 
interpretability.

To address these issues, the clustering approach assumes that each voter $i$ belongs to one of $K$ "clusters", or latent groupings. We denote this membership as a random variable, $Z_{i}$, and index clusters by $k \in\{1, \ldots, K\}$. Importantly, although different individuals may belong to different clusters, there is no differentiation of clusters within an individual even across different offices.

It then posits the following model of vote choice that incorporates our two key parameters of interest. The prevalence of cluster $k$ in the population by $\pi_{k}$, where $\boldsymbol{\pi}$ is a $K$-length proportion that sums to $1\left(\sum_{k=1}^{K} \pi_{k}=1\right)$, so that

$$
Z_{i} \sim \operatorname{Categorical}(\boldsymbol{\pi})
$$

Next, to characterize the each cluster $\mu_{j k \ell}$ represents the latent propensity for any member of cluster $k$ to vote for a particular option $\ell$ in office $j$, so for a given cluster and given office, $\sum_{\ell^{\prime}=0}^{L} \mu_{j k \ell^{\prime}}=1$.

$$
\operatorname{Pr}\left(Y_{i j}=\ell \mid Z_{i}=k\right)=\mu_{k j \ell}
$$

For example, a political campaign may be interested in the size of the swing voter bloc and how likely that bloc is to split their ticket for a particular candidate in office $j$. In this case, if we had estimated two clusters, and set aside the first cluster for staunch partisans and the rest for potential swing voters, we would want to know the quantity $\pi_{2}$ (the size of the bloc) and $\mu_{2, j \text {,split }}$

This modeling choice maps to a theoretical notion that ticket splitting is a probabilistic function of being a swing voter. This stands in contrast to existing approaches, which is more deterministic. Although splitting one's ticket may be a sufficient indicator of being a swing voter, it is not a necessary condition because a swing voter 
that is indifferent to either party should have roughly equal probability of choosing one candidate over the other (Larcinese et al. 2013).

\subsection{Estimation Strategy}

Our goal is to estimate the unobserved parameters $\boldsymbol{\pi}, \boldsymbol{\mu}$ that is most consistent with the data that we do observe, i.e. the vote choice matrix Y. To do so, we must assume the full data generation model as a function of the data and parameters. Once we assume that the probability of a particular vote is independent across offices within the same cluster, we can express the likelihood as a product of $J$ factors:

$$
\operatorname{Pr}\left(\mathbf{Y}_{i} \mid Z_{i}=k, \boldsymbol{\pi}\right)=\prod_{j=1}^{J} \text { Categorical }\left(Y_{i j} \mid \boldsymbol{\mu}_{k}\right)=\prod_{j=1}^{J} \prod_{\ell=0}^{L} \mu_{k j \ell}^{\mathbf{1}\left(Y_{i j}=\ell\right)}
$$

which is similar to a standard multinomial regression except that we observe $J$ data points for each voter instead of one, and that we actually do not observe the conditioning variable $Z_{i}$. The independence assumption may at first seem unrealistic: a voter's propensity to vote for a Democrat in one office is surely dependent with his propensity to vote for a Democrat in the next. Note that we assume independence only within a cluster. In other words, this model allows for the dependence across offices by averaging over clusters.

I derive an Expectation Maximization (EM) algorithm to quickly estimate the parameters (details left to the Appendix). Because clusters are latent, traditional Maximum Likelihood Estimation (MLE) proves intractable. EM is a well-established iterative procedure that is guaranteed to find a (at least local) MLE. ${ }^{3}$ In political science,

3 It is well known that the EM algorithm can get "stuck" in local (as opposed to global) maxima depending on the starting values. Following Linzer and Lewis (2011), I initialize parameters with a simple $k$-means clustering on binarized data, and run 10 versions of the same model but with different random seeds for the initialization. Then, I pick the model that has the highest log likelihood. 
EM has been successfully employed in ideal point estimation (Imai et al. 2016). The statistical insight of EM is that even though we do not know the cluster assignment $Z_{i}$, we can temporarily replace it with posterior expectation $\mathbb{E}\left[Z_{i}=k \mid \mathbf{Y}_{i}\right]$ (the E-step), estimate the MLE of the parameters assuming those are values (the M-step), and cycle through the same steps. The observed likelihood is re-computed at each step as a function of the parameter estimates, and we stop the model once it stops increasing by more than a pre-set threshold.

The EM approach to cluster modeling is also employed in the poLCA (polytomous variable Latent Class Analysis) package by Linzer and Lewis (2011), but the model and package I have derived has several additional features. As I show below, I allow for varying choice sets to model uncontested races instead of listwise deleting voters facing an uncontested race. By incorporating more extensive $\mathrm{C}++$ backends to the computation and fast algorithms for intermediate steps (Yamauchi 2020), my package is also faster by about a factor of ten than existing software in large datasets (See the Appendix). Finally, I visualize the estimated parameters in a more intuitive and interpretable format.

How does the clustering approach differ from ideal point estimation, which is another common way political scientists have summarized large voting data (Lewis 2001; Gerber and Lewis 2004)? Ideal point estimation often reduces to solving a very similar likelihood function as clustering, but instead of setting the probability $\mu$ as the quantity of interest, they posit that decisions are made according to a one-dimensional spatial voting model and estimates voting preferences on a continuum, rather than as separate blocs. The clustering approach can be thought of as trading away a parsimonious one-dimensional model for a more flexible approach to classify individuals that does not rely on a spatial model of vote choice. Users can choose the numbers of clusters to estimate, and the clusters are not restricted to be placed on a particular coordinate 
space.

\subsection{Additional Features of the Clustering Model}

So far, the clustering model discussed here follows the canonical model of finite mixtures for categorical outcomes. Several additional features are relevant for analyzing vote data.

Respondent Level Covariates It is natural to posit that certain demographic groups are systematically more likely to be in particular clusters. Clustering models can incorporate such auxiliary data about the respondents in a straightforward manner by modeling the cluster assignment as a function of covariates. Suppose we have an indicator for whether every voter is an ideological moderate. The spatial voting model would predict that this indicator to positively correlate with assignment into a cluster that tends to have high rates of ticket splitting.

Respondent-level covariates like these can be incorporated into the EM algorithm's M-step by regressing the expectation of cluster assignment on covariates in what is essentially a weighted multinomial logit model. ${ }^{4}$ A model with and without such covariates should produce roughly similar cluster assignments because we still infer these from the votes. But incorporating covariates can help stabilize the algorithm, and the values of the coefficients $\gamma$ provide useful substantive information for interpreting cluster membership. The ECM algorithm implemented by Yamauchi (2020) makes this step fast enough to be repeated at each step in the main EM loop.

4 Formally, we replace 1 with $\pi_{i k}=\frac{\exp \left(\boldsymbol{X}_{i}^{\top} \boldsymbol{\gamma}_{k}\right)}{\sum_{k^{\prime}=1}^{K} \exp \left(\boldsymbol{X}_{i}^{\top} \boldsymbol{\gamma}_{k}\right)}$, where $\boldsymbol{X}$ is a $N \times P$ matrix and $\boldsymbol{\gamma}_{k}$ are $P+1$ coefficients and an intercept. As this shows, this requires we let $\boldsymbol{\pi}$ be a matrix with $N$ rows. When summarizing the data in subsequent analyses, I take the average mixing proportion for each cluster as an aggregate measure. 
Varying Choice Sets Many elections for state and local offices are uncontested, which means that a voter still makes a choice, but from a limited menu of options. These different settings require modelling varying choice sets. While existing discrete clustering models rule out this possibility and therefore require analysts to drop data that includes varying choice sets, I model these separately, with an independence of irrelevant alternatives (IIA) assumption to share information and parameter values across observations.

The added complication is that the vote choice probability must now be modeled as a function of data that varies by the choice set. Let $\mathcal{Y}_{i j}$ denote the set of values that are available to voter $i$ in option $j$. Such information would be clear from the candidate filings in that district, and so are directly observed. We then posit that the choice probability is generated from a ratio that is relative to the available choices for a given voter, as in a standard multinomial logit. ${ }^{5}$ To identify the model I set the baseline for abstention to be 0 . To estimate these values quickly, I use a BFGS optimization of the likelihood.

\subsection{Limitations and Issues of Interpretation}

Before moving to empirical analyses, I note important implications of this modeling approach that some may see as limitations. Clustering algorithms, though widespread in computer science and other fields such as marketing and psychology, is clearly less used in political science or economics and so it is important to consider why.

First, the type of data this method can handle are restricted to datasets where (i) the outcome measurement is categorical and (ii) come from the roughly same choice set

\footnotetext{
5 Formally, we parameterize equation 2 as $\operatorname{Pr}\left(Y_{i j}=\ell \mid Z_{i}=k\right)=\frac{\exp \left(\psi_{k j \ell}\right)}{\sum_{\ell^{\prime} \in \mathcal{Y}_{i j}} \exp \left(\psi_{k j \ell^{\prime}}\right)}$ where $\psi_{\ell}$ is a
} scalar that represents the intensity of preference for option $\ell \in\{1,2\}$ relative to $\ell=0$ (abstention). 
across offices. ${ }^{6}$ For example, it would not be possible to analyze a set of variables that includes vote choice and numerical responses. Similarly the model also cannot handle ballot data where some variables are partisan offices (Republican, Democrat) while others are nonpartisan offices or referendums (Yes, No), unless the analyst is willing to assume that voting "Yes" on a particular referendum represents the same underlying event as voting for a Republican or Democrat. For such cases of mixed ballots that violate (ii), an ideal point model will be more appropriate because it targets a single dimensional preference estimate and maps different votes to a single space. For cases that violate (i), one must turn to other clustering methods like $k$-means for datasets with only continuous outcomes, and more involved models for a mix of continuous and categorical outcomes.

On a more important theoretical point, clustering algorithms almost always require the user to pre-determine the number of clusters $K$ to model from the data. Therefore, one might worry that substantive findings from data may change wildly by the number of clusters. There do exist methods to pick the optimal number of clusters based on measures of model fit (Fraley and Raftery 1998) - essentially functions of the observed likelihood attempting to account for overfitting.

But we do not need to believe that there is one "correct" number of clusters the analyst has to identify in order for clustering analyses to be useful. As Broockman (2016, p. 207) argues, voter's preferences are likely formed by hundreds of small issue "dimensions", even though each one may not incrementally improve model fit. Whether one models 2 clusters or 3 clusters from the data is not a claim about the analyst believing that 2 or 3 dimensions is enough to explain voting behavior. Instead, this method can be thought of as a principled way to summarize information and characterize proto-

6 While I allow for varying choice sets, a better name for this commonly used term is a "limited choice set," i.e. one of the options being missing. 
typical voting patterns given the user's chosen level of granularity. Substantive theory, rather than only a statistical information criterion, should guide the choice of the number of clusters.

A related concern about unsupervised learning methods is that interpretation of each cluster is arbitrary. Examining the correlation of covariates with estimated cluster assignment is a useful way to uncover some interpretation. But the analyst must also bring some of their own substantive knowledge for this clustering algorithm to be useful. Indeed, model output should not be interpreted as anything more than as a summary of the data based on a simple probabilistic model of vote choice. In the same way that there is rarely a single "correct" number of clusters, it is actually reasonable to pick the number of clusters so that it reveals clusters whose estimated parameters $\boldsymbol{\mu}$ match the theoretical quantity of interest. In my applications, the main quantities of interest are the size and voting patterns of swing voters, which the model parameters directly target.

Of course, one must start somewhere. One reasonable initial choice is $K=2$, which is the simplest case and also has parallels to many theories like the black-white model or core-and-periphery. Or, one can start by setting the number of clusters to the number of response options there are. This allows the data to cluster into homogeneous response specific clusters, if that is the underlying pattern. In the context of the core vs. swing model, one might posit that voters can be partitioned into swing voters who split their ticket regardless of the office, abstention voters who undervote regardless of the office, and so on. This is a useful null hypothesis that I test on the data, and ultimately reject. 


\section{Application to Cast Vote Records}

The clustering algorithm is well suited to glean patterns from large datasets of anonymous ballots. I first illustrate the insights from the clustering approach by analyzing ballots from the Florida 2000 election, which were originally analyzed in an ideal point framework.

Both political scientists and election administrators use ballot data to understand voter behavior and ballot design, but the high-dimensional and large- $N$ nature of these datasets make analysis challenging. For example, early work by Jeff Lewis and colleagues have analyzed ballots from Florida, South Carolina and Los Angeles country to study the political preference of Ralph Nader voters, election anomalies, and the multidimensional structure of voter's preferences over national and local issues (Herron and Lewis 2007; Bafumi et al. 2012; Gerber and Lewis 2004). More recently, Kuriwaki (2019) has used this data to ticket splitting behavior across the long ballot, including in offices where surveys cannot poll, and Morse (2019) collects ballots from the Florida 2018 election to study voter behavior for criminal justice and voting rights policies. Finally, cast vote records have become integral for transparent election administration, in particular for the implementation of risk limiting audits which require that state secretaries of state can readily sample from the population of ballots (Stark 2008; McCarthy et al. 2018; for a review see Kuriwaki 2020).

Herron and Lewis (2007) coded the ballot punch cards from over 3 million ballots from ten large counties in Florida, guided by the question: Did Ralph Nader spoil Gore's victory in Florida - and thus the presidency — by drawing leftist voters who would have supported voted for Gore in the absence of a third party candidate? Surveys data are too sparse and unreliable to estimate the preferences of a subpopulation like Nader voters, so the authors turn to cast vote records to analyze the down-ballot voting 
patterns and estimate ideal points for each voting pattern. By imposing a spatial model with candidate valence on the data, the authors are able to predict whether Nader voters were more proximate to Gore than for Bush.

In this application, I use the author's replication data but apply the clustering algorithm instead of an ideal point model to answer the main substantive question of this paper: what proportion of voters could be considered swing voters, and how likely were they to split their ticket? I leverage the joint distribution of votes available in the long ballot and analyze one large county - Palm Beach County — that had many partisan offices on the 2000 ballot. This subset still contains 300,000 votes, more than any survey of the entire state. I use almost all the partisan races on the ballot: both chambers of Congress, two statewide cabinet positions (Education Commissioner and Treasurer), the public defender for the 15th circuit court of Florida, and three county-wide executive offices (Clerk of the Court, Sheriff, and Tax collector).

I use voter's choice in the Presidential race as the reference category for coding whether a voter votes for a straight or split ticket. Because this outcome is not well defined for voters who chose a third party candidate in the Presidential race, I drop these from consideration so that the choice set to is straight ticket vote, split ticket vote, third party vote, or undervote (abstention)\}. ${ }^{7}$ I cannot determine the congressional district of those with an undervote in that office, so I further subset to the vast majority of voters who cast a vote in one of the four congressional districts in the county.

Figure 1 panel (A) shows the estimated parameters for the size of each cluster $(\boldsymbol{\pi})$ and the latent voting probabilities $\boldsymbol{\mu}$, faceted by the 4 clusters that partition the electorate. Each of the three panels represent one run of the clustering algorithm. The

7 In this county, none of the offices I mention were uncontested. I analyze the case of uncontested House races in the next application. 
Figure 1: Clusters of Voting Profiles in Palm Beach County Florida, 2000

(A) All Voters for Gore or Bush

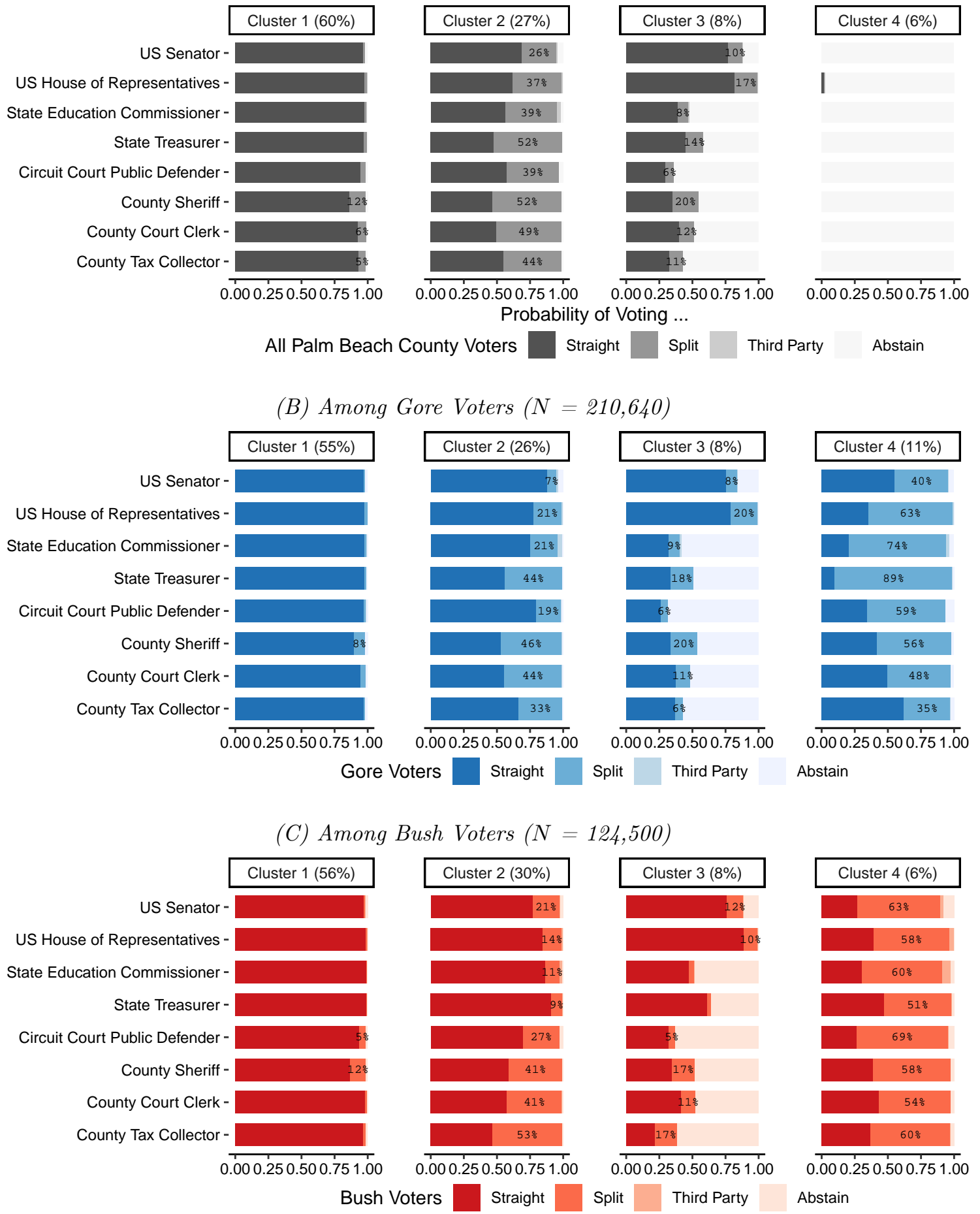

Note: Plots Estimates from the Clustering Algorithm, where one panel is from one model. The length of each bar indicates the estimated value of $\mu_{k j \ell}$, or the probability voters in cluster $k$ votes for option $\ell \in\{$ abstain, straight, split, other $\}$ in office $j$. 
length of each segment represent the vote choice quantities $\boldsymbol{\mu}$ for a particular office and cluster of voters. I initially chose four clusters because there are four possible response categories, as reasoned in the previous section. Because clusters have no inherent ordering in the statistical model, throughout this paper I number the clusters by size the estimates of $\boldsymbol{\pi}$ : the cluster estimated to capture the most number of voters is called cluster 1, the second largest as cluster 2, and so on. I do not use covariate data such as congressional district in this current application, but I conduct separate analysis by the Presidential vote choice covariate in panel (B) and (C).

The estimated parameters offer a straightforward summary of a vast amount of high dimensional data. First we see that about 55 to 60 percent of Bush and Gore voters are straight ticket voters (cluster 1), who vote for the same party's candidates (Democrats in Panel (B) and Republicans in Panel (C)). Next, about 26 to 30 percent of voters have a decent probability of crossing the party line. Interestingly, this group in cluster 2 is more likely to split further down the ballot, i.e. in state and local offices. This is consistent with the argument in Kuriwaki (2019) that although partisan cues may be relatively stronger in low information environments (Peterson 2017), there is also a strong incumbency advantage and valence differential between candidates in state and local elections.

Next, cluster 3 represents about 8 percent of the population and are mostly characterized by undervotes. ${ }^{8}$ There is a notable office-specific pattern here, too. These voters appear to abstain in state and local offices, but still vote straight ticket for Congress. Cluster 4 also has high levels of ticket splitting but is the smallest group among Bush voters. In fact, when both of the subgroups are stacked together in Panel

8 Among Gore voters, I flip the numbering between cluster 3 and 4 so that each clustering's voting patterns are similar to their counterparts in the other panels. This violates the rule for numbering clusters by estimated size, but here the sizes are similar enough that I opt from the gains in comparability. 


\section{Figure 2: Swing Voters by Presidential Support}

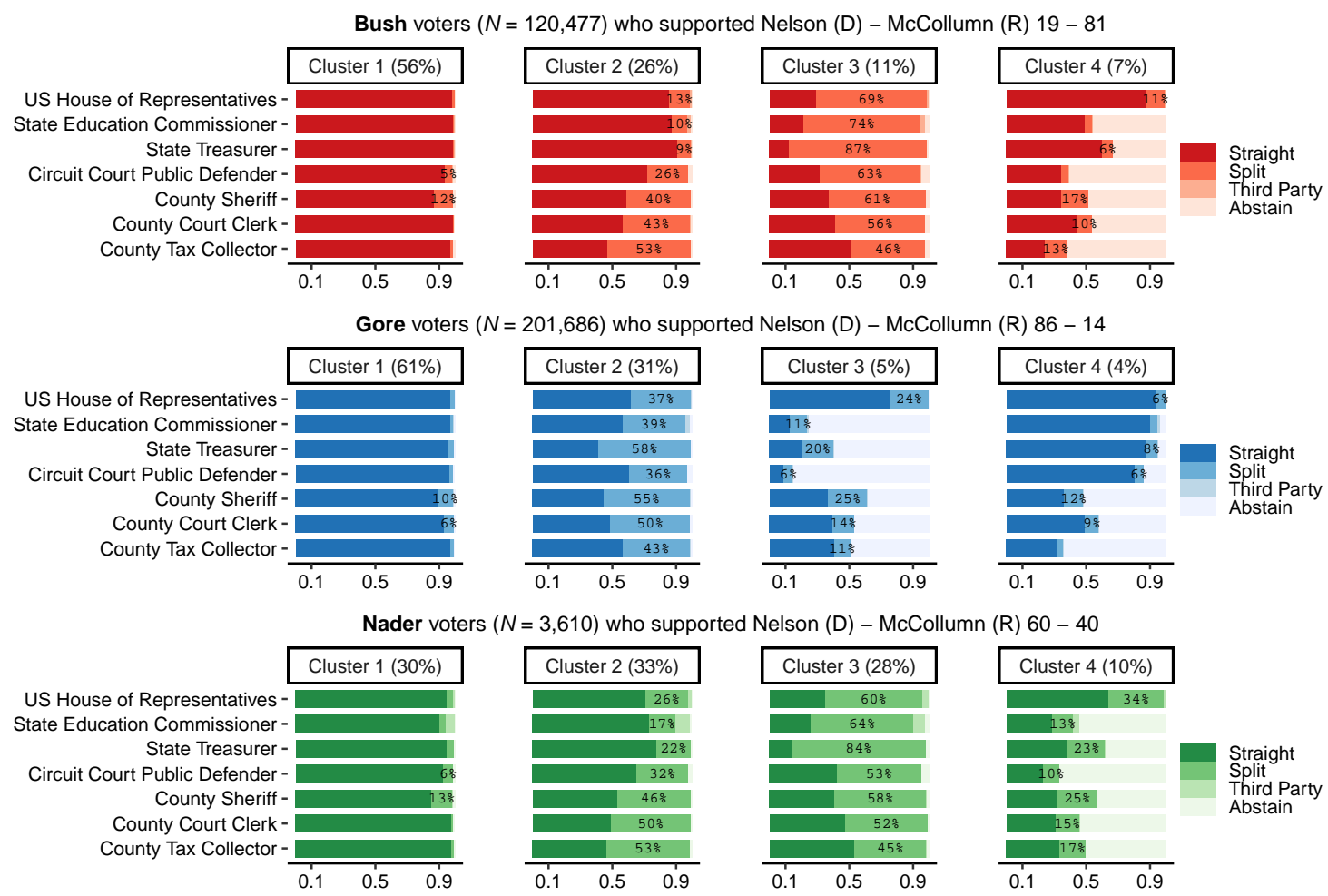

Note: Figure format follows Figure 1 in showing Palm Beach county voters but recodes the reference category to the US Senate race, and includes Nader voters who voted for a Republican or Democratic Senate candidate.

(A), an all-undervote cluster arises as the fourth cluster.

The original question in Herron and Lewis (2007) was to study how similar preferences of Ralph Nader voters were to Gore voters, which we can also re-assess with the current clustering approach. In Figure 1, I had dropped Nader voters entirely from the analysis because our outcome was coded with reference to the two-party vote in the Presidential race, but in Figure 2 I recode the outcomes with reference to the US Senate race between Nelson (D) and McColumn (R), and then run separate clustering algorithms by Presidential vote choice among Bush, Gore, and Nader voters. ${ }^{9}$ Nader

9 Therefore, one note of implication here is that now the voters in each panel are a mix of Nelson and McColumn voters. 
voters exhibit different patterns of ticket splitting. Cluster 3, which is more likely to split their ticket than stick to their Senate choice, comprises 28 percent of Nader voters but only 11 percent of Bush voters and 8 percent of Gore voters. These findings are roughly consistent with the original paper's findings based on a IRT model, namely that Nader voters were not in fact predominantly "left" of Gore, and so it is not clear if Nader handed the Presidency to Bush by running. For example, Nader voters only supported the Democratic Senate candidate 60 - 40.

The picture of the electorate that emerges from these analyses is one in which 60 percent were party loyalists and about 5 percent roll off, but where a quarter of the vote can be considered as a reasonable swing bloc. Further, the clustering model's parameter estimates in both cluster 2 and cluster 3 help refute the null hypothesis that voter types vote straight regardless of the office. While a majority of voters were consistently straight partisans, the rest vote differently, with a particular difference between Congressional, state, and local offices.

\section{Application to Survey Data}

In my second application, I turn to the 2018 midterm election using survey data from the Cooperative Congressional Election Study (CCES). Survey data is limited by its small sample size and potential risk of measurement error, but it provides comparable data across states and a rich set of individual-level covariates that anonymous ballot images lack. Moreover, surveys have more wide-ranging uses in social science research and polling. Like the ballot data, the clustering algorithm can be applied to respondents who answer a series of questions that all roughly draw from the same set of categorical outcomes.

As illustration, I use the midterm ballot in 2018. Midterm elections are an interesting case for the study of the swing voter because many state-level offices are 
Table 1: States Analyzed in the 2018 CCES

\begin{tabular}{rccccr}
\hline & \multicolumn{2}{c}{ Incumbent (running for re-election) } & & \\
\cline { 2 - 4 } State & US Senator & Governor & Attorney Gen. & CDs & CCES $n$ \\
\hline Massachusetts & Warren (D) & Baker (R) & Healey (D) & $7(5)$ & 688 \\
Maryland & Cardin (D) & Hogan (R) & Fosh (D) & $8(8)$ & 663 \\
Texas & Cruz (R) & Abbott (R) & Paxton (R) & $36(32)$ & 1,128 \\
New York & Gillibrand (D) & Cuomo (D) & & $27(21)$ & 1,990 \\
Florida & Nelson (D) & & $27(22)$ & 2,817 \\
Michigan & Stabenow (D) & & $14(13)$ & 1,156 \\
Wisconsin & Baldwin (D) & Walker (R) & Schimel (R) & $8(7)$ & 757 \\
Ohio & Brown (D) & & & $16(16)$ & 1,555 \\
Arizona & & Ducey (R) & Brnovich (R) & $9(8)$ & 1,051 \\
Minnesota & Klobuchar (D) & & & $8(8)$ & 652 \\
\hline
\end{tabular}

Note: The ten largest states (in terms of CCES sample size) that held contested elections for US Senate, Governor, and Attorney General in 2018. Incumbent columns show the name and party of the incumbent if running for re-election; open (but contested) seats are left blank. CDs indicate the number of US House districts in the state, with the number of contested districts in parentheses. States are ordered as in Figure 3.

elected in midterm years. I first gather the post-election wave's self-report responses for Congress, Governor, and State Attorney General in ten of the largest states where those offices were on the ballot, shown in Table 1. Later, I also compare the results from the 2014 CCES, a year in which many Republicans flipped seats, in contrast to 2018 which saw a Democratic resurgence.

I code the respondent's vote choice as straight / split vote with the voter's partisan self identification as the reference category, instead of presidential vote choice as in the Palm Beach example. I drop pure independents because the notion of ticket splitting is not well defined for this group, and make inferences about the voters who identify with or lean towards one party or the other. Therefore, the sample definition is slightly different from the previous example, but still captures more than 80 percent of the electorate sampled. I model the uncontested races in the US House using the IIA assumption previously discussed. I also model cluster assignment with the respondent 
covariates for Democrat, White, high news interest, identification as an ideological moderate. I fit clusterCVR using three $\operatorname{clusters}^{10}$, generating 10 replicates and picking the model with the highest log likelihood.

Figure 3 shows the final estimated cluster sizes and cluster characteristics, by state and cluster, in the same fashion as before. To understand which types of partisans and demographic groups were more likely to be in particular clusters, we plot the estimated coefficients $(\gamma$ in footnote 4$)$. States in the figure are ordered by the estimated size of the first cluster, which is clearly the cluster for straight ticket voters as it was in the Palm Beach county example.

Across all states, we find that the majority of the electorate are straight ticket voters, who are all but certain to vote for the same candidates as their party. But the proportion of these voters ranges from close to 95 percent in Minnesota to nearly 60 percent in Massachusetts. The remaining two clusters appear to contain various types of ticket splitters.

Interestingly, the pattern of ticket-splitting is state and office specific. Figure 3 shows that states where the core partisan bloc is smallest are Massachusetts, Maryland, and Texas. In all three, the Governor vote stands out as in the second cluster, approaching 30 percent of the voters in Massachusetts. As the table of candidates in Table 1 shows, in all three states a Republican incumbent ran for re-election. The coefficients on the voter's Democratic partisan identification predicting cluster membership (Figure 4) indicate that in Massachusetts and Maryland, it was Democrats who split their ticket (for Baker and Hogan, respectively), whereas in Texas, it was Republicans who split their ticket (against Abbott). In Wisconsin, the only other state in Figure 3 in which a Republican Governor ran for re-election, there is no clear Governor-specific

10 Estimating four clusters recovered similar findings for the swing voter bloc, but was sensitive due to much smaller samples in certain states compared to the ballot data example. 
Figure 3: Clusters in 2018

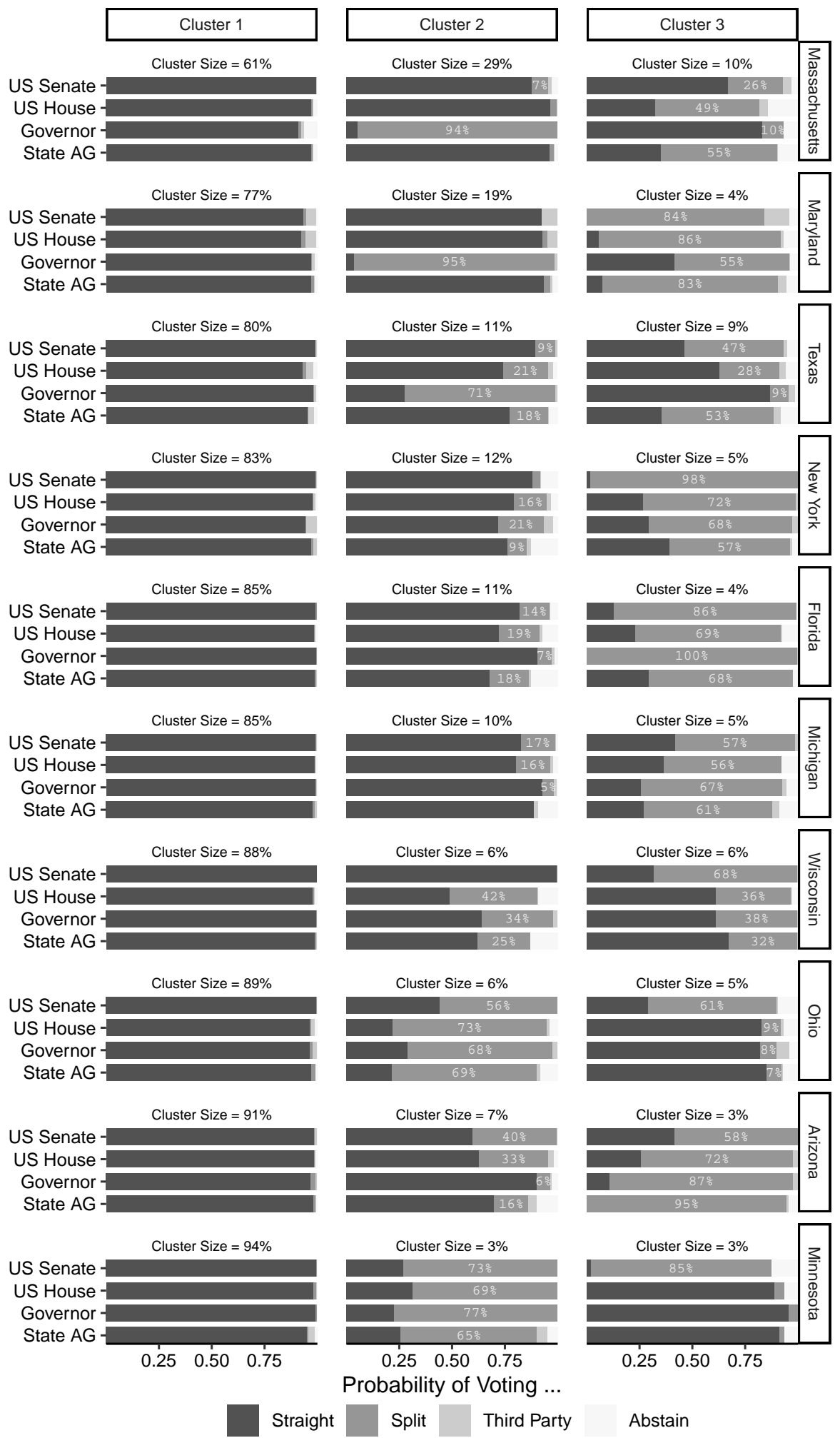

Note: Each facet shows the size and estimated latent voting patterns of an estimated cluster, for a given state and given cluster. Data: CCES 2018. 


\section{Figure 4: Demographic Predictors of Cluster Membership}

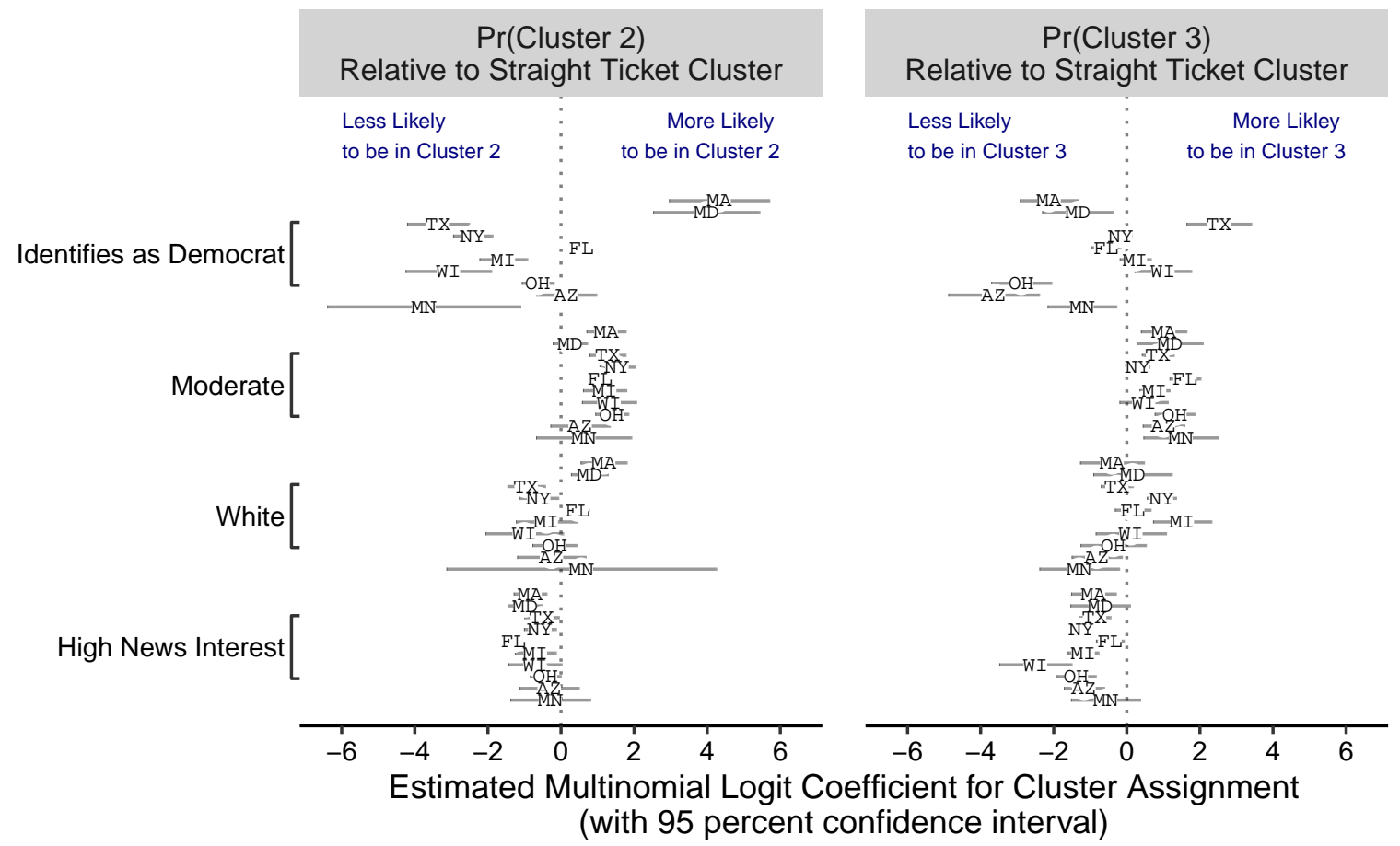

Note: Each point is a coefficient estimate for predicting cluster membership estimated within the clustering process. In all states, moderates and low news interest voters are more likely to be in the two smaller clusters, but the correlation by party differs starkly by state. All four predictors are coded to be binary indicators. Letters correspond to the state being estimated and one clustering algorithm is estimated per state. Cluster numbers correspond to those in Figure 3. Data: CCES 2018.

bloc, but both Republicans (cluster 2) and Democrats (cluster 3) were most equally likely to cross party lines in their vote for Governor. In New York state, where Cuomo ran for re-election there is no clear Governor vote. That Massachusetts and Maryland to the top in the Governor vote is not necessarily surprising, given that Baker and Hogan were the nation's two most popular Governors in the summer leading up to the election, and won with large margins. ${ }^{11}$ The clustering estimates, however, add more insight than these standard statistics because they summarize the survey data into voter prototypes: it shows that Democrats supporting Baker and Hogan still voted for

11 "America's Most and Least Popular Governors". Morning Consult Poll, July 25, 2018. https: //perma.cc/2XYN-NJZ7 
Democratic candidates in other offices.

We also see clear, if smaller, blocs of ticket splitting for incumbent Senators in cluster 3 of Ohio (Brown) and Minnesota (Klobuchar). In both of these states, Republicans were more likely to be in the cluster that were more likely to ticket-split, indicating that they voted for the Democratic incumbents. We do not see similar blocs of pro-incumbent Senator voting blocs in Massachusetts (Warren), Maryland (Cardin), Texas (Cruz), and Michigan (Stabenow). These patterns across different candidates of the same offices are consistent with the notion that popular incumbents tend to attract ticket splitting votes.

It may be the case that simple cross-state comparisons are confounded by fixed characteristics of the state's electorate. Therefore as a final step, I repeat this analysis 4 years in prior, in states where the same offices (but usually not the same candidates) were on the ballot. Three states out of the ten shown in Figure 3 meet that criteria: Massachusetts, Texas, and Michigan. I ran the clustering model on similar 2014 CCES data, and show the estimated model parameters side by side in Figure 5.

The Governor-specific ticket splitting found in Massachusetts and Texas in 2018 are not found in 2014, even though the same Republican candidate was on the ballot. In 2014, few voters were classifiable as swing, but in 2018 Baker netted more votes from Democrats and won 67 - 33 while Abbott suffered from Republican defections (but pulled of re-election despite it, winning 56 - 43). In Michigan, the composition of straight and split ticket voting blocs did not change significantly.

\section{Conclusion}

In this paper I introduced a clustering model for election observers to draw insights from large- $N$, high-dimensional data. I outlined a simple model of vote choice for partisan races on the long ballot, and offered some guidance on how data analysts 
Figure 5: Clusters between 2014 and 2018

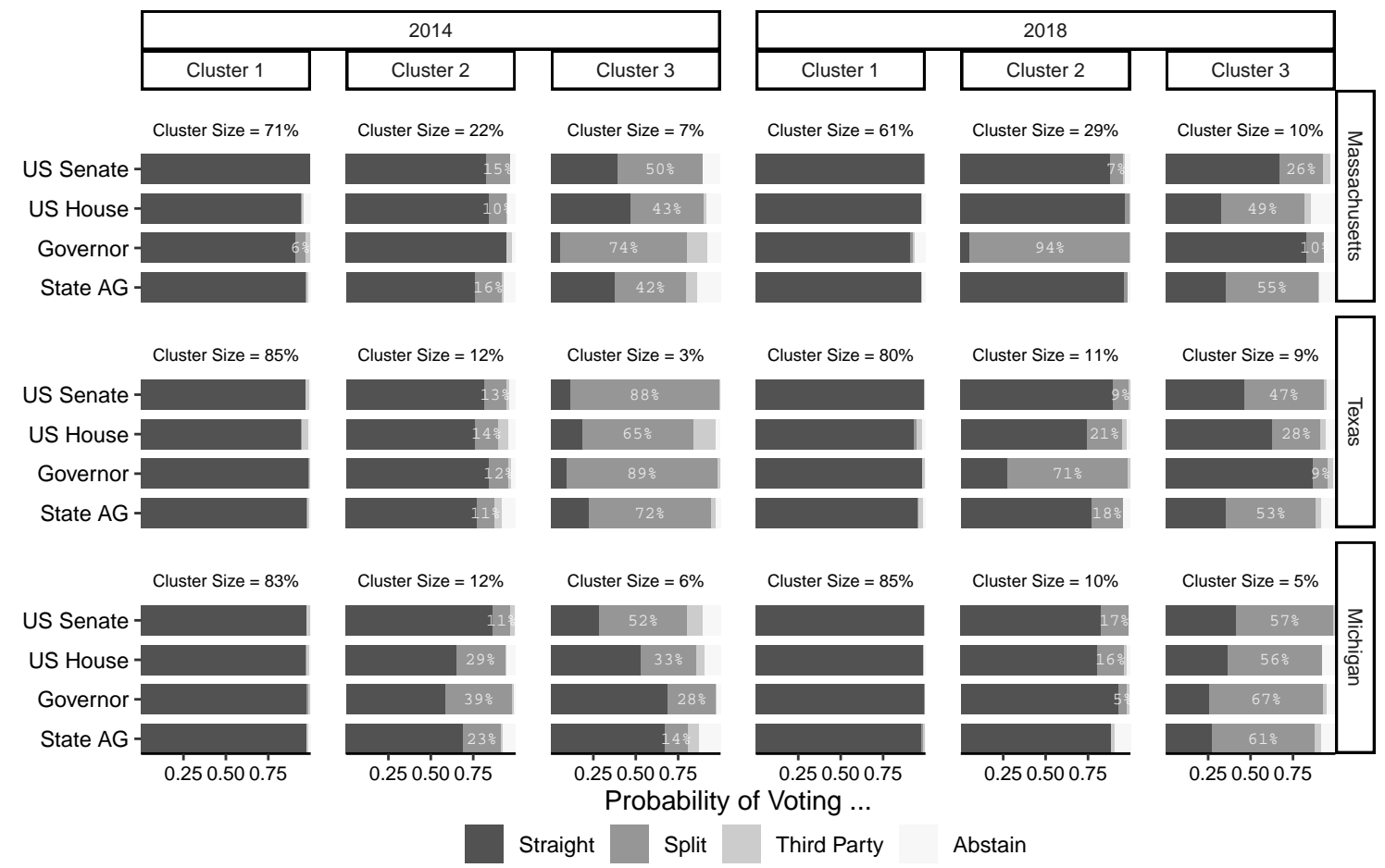

Note: A separate analysis is run for all states which held a contested US Senate, Governor, and State Attorney General race in both 2014 and 2018.

should interpret the model estimates of latent quantities.

In an application to the crucial vote in Palm Beach County in Florida, the clustering method found that a majority of strong partisans (although not a super-majority), about 20-30 percent of potential swing voters who split disproportionately in state and local offices, and about 8 percent of rolloff voters who still voted for their members of Congress. In the second application to survey data in 2018, the method reveals about that about 80-90 percent of votes who identify with one party are straight ticket voters. But popular Governors and some popular Senators drawing voters across the party line and effectively forming blocs that deliver their re-election.

The statistical model used here can benefit from several more additions in the future. First, one can include choice-specific covariates such as candidate ideology, 
candidate incumbency, and candidate gender, directly into the estimation. These coefficients are widely analyzed in multinomial logit models of consumer choice but a faster algorithm to solve such models must be derived to incorporate them into a clustering algorithm. Modeling district-specific characteristics as random effects by positing that they are drawn from a common distribution is another possible feature to add to the modeling process, although estimation of such models in multinomial regression also remains an active area of statistics research (Linderman et al. 2015).

I have shown here that a fairly straightforward application of a clustering model can be applied to illuminate patterns and identify groups from complex voting data. As I have discussed, these tools should not be a substitute for substantive theorizing and interpretation, but they can notably facilitate discovery, provide a more principled measurement of size and voting propensity, and improve theory building by providing data-based guidance. 


\section{References}

Ahlquist, John S. and Christian Breunig (2012). "Model-based clustering and typologies in the social sciences". Political Analysis 20(1), 92-112. DOI: 10.1093/pan/mpr039.

Bafumi, Joseph, Michael C. Herron, Seth J. Hill, and Jeffrey B. Lewis (2012). "Alvin Greene? Who? How Did He Win the United States Senate Nomination in South Carolina?" Election Law Journal 11(4), 358-379. DOI: 10.1089/el j .2011.0137.

Beck, Paul Allen, Lawrence Baum, Aage R. Clausen, and Charles E. Smith (1992). "Patterns and Sources of Ticket Splitting in Subpresidential Voting". American Political Science Review 86(4), 916-928. DOI: 10.2307/1964344.

Broockman, David E. (2016). "Approaches to Studying Policy Representation". Legislative Studies Quarterly 41(1), 181-215. DOI: 10.1111/lsq.12110.

Burden, Barry C. and David C. Kimball (2002). Why Americans Split Their Tickets: Campaigns, Competition, and Divided Government. University of Michigan Press.

Campbell, Angus (1960). "Surge and Decline: A Study of Electoral Change". Public Opinion Quarterly 24, 397-418.

Carnes, Nicholas and Noam Lupu (2020). "The White Working Class and the 2016 Election". Perspectives on Politics. DOI: 10.1017/S1537592720001267.

Carroll, Susan J. (1999). "The Disempowerment of the Gender Gap: Soccer Moms and the 1996 Elections". PS: Political Science and Politics 32(1), 7. DOI: 10.2307/ 420743.

Cohn, Nate (2019). A Sliver of the Electorate Could Decide 2020. Here's What These Voters Want. URL: https://nyti.ms/2NEC2sY.

Dubin, Jeffrey A. and Elisabeth R. Gerber (1992). "Patterns of Voting on Ballot Propositions: A Mixture Model of Voter Types".

Estevez-Abe, Margarita, Torben Iversen, and David Soskice (2010). "Social Protection and the Formation of Skills-A Reinterpretation of the Welfare State". 95th American Political Association Meeting, 1-80. DOI: 10.1093/0199247757.001.0001.

Fiske, Susan T., Amy J. C. Cuddy, Peter Glick, and Jun Xu (2002). "A model of (often mixed) stereotype content: Competence and warmth respectively follow from perceived status and competition." Journal of Personality and Social Psychology 82(6), 878-902. DOI: 10 .1037//0022 - 3514 . 82 .6 . 878. URL: http : //doi . apa .org/ getdoi. cfm?doi=10.1037/0022-3514.82 -6.878. 
Fraley, Chirs and Adrian E. Raftery (1998). "How Many Clusters? Which Clustering Method? Answers Via Model-Based Cluster Analysis". The Computer Journal 41(8), 578-588. DOI: 10.1093/comjnl/41.8.578.

Gerber, Elisabeth R. and Jeffrey B. Lewis (2004). "Beyond the Median: Voter Preferences, District Heterogeneity, and Political Representation". Journal of Political Economy 112(6), 1364-1383. DOI: 10.1086/424737.

Goplerud, Max (2019). "A Multinomial Framework for Ideal Point Estimation". Political Analysis 27(1), 69-89. DOI: 10.1017/pan.2018.31.

Grimmer, Justin and Gary King (2011). "General purpose computer-assisted clustering and conceptualization". Proceedings of the National Academy of Sciences 108(7), 2643-2650. DOI: 10.1073/pnas. 1018067108.

Grimmer, Justin and William Marble (2019). "Who Put Trump in the White House? Explaining the Contribution of Voting Blocs to Trump's Victory", Working Paper. URL: https://williammarble.co/docs/vb.pdf.

Herron, Michael C. and Jeffrey B. Lewis (2007). "Did Ralph Nader Spoil a Gore presidency? A Ballot-level Study of Green and Reform Party Voters in the 2000 Presidential Election". Quarterly Journal of Political Science 2(3), 205-226. DOI: $10.1561 / 100.00005039$.

Hill, Jennifer L. and Hanspeter Kriesi (2001). "Classification by Opinion-Changing Behavior: A Mixture Model Approach". Political Analysis 9(4), 301-324. DOI: 10. 1093/oxfordjournals . pan . a004872.

Imai, Kosuke, James Lo, and Jonathan Olmsted (2016). "Fast Estimation of Ideal Points with Massive Data". American Political Science Review 110(4), 631-656.

Key, V. O. (1966). The Responsible Electorate: Rationality in Presidential Voting: 19361960. Harvard University Press.

Kuriwaki, Shiro (2019). "Party Loyalty on the Long Ballot: Is Ticket Splitting More Prevalent in State and Local Elections?" URL: http://doi.org/10.31235/osf.io/ bvgz3.

Kuriwaki, Shiro (2020). "The Administration of Cast Vote Records in U.S. States". URL: https://bit. ly/3dxcoTD.

Larcinese, Valentino, James M. Snyder, and Cecilia Testa (2013). "Testing models of distributive politics using exit polls to measure voters' preferences and partisanship". British Journal of Political Science 43(4), 1-31. DOI: 10. 1017/S0007123412000245. 
Lewis, Jeffrey B. (2001). "Estimating Voter Preference Distributions from IndividualLevel Voting Data". Political Analysis 9(3), 275-297. DOI: 10.1093/polana/9.3.275.

Linderman, Scott W., Matthew J. Johnson, and Ryan P. Adams (2015). "Dependent multinomial models made easy: Stick breaking with the Pólya-gamma augmentation". arXiv: arXiv: 1506.05843v1.

Linzer, Drew A. and Jefrey B. Lewis (2011). "poLCA: An R Package for Polytomous Variable Latent Class Analysis". Journal of Statistical Software 42(10), 1-18. DOI: 10. 4018/978-1 - 4666- 2931- 8. ch001.

McCarthy, John, Neal McBurnett, Harvie Branscomb, Ron Rivest, and Philip Stark (2018). Preserving Anonymity of Cast Vote Record. Tech. rep., 1-11. URL: http: //bit. ly/2LcuCOQ.

Morse, Michael (2019). Opinion: Amendment 4 Requires Addressing the Criminalization of Poverty. URL: http://bit.ly/2LJHNq3.

Müllner, Daniel (2013). "fastcluster: Fast hierarchical, agglomerative clustering routines for R and Python". Journal of Statistical Software 53(9), 1-18. DOI: 10.18637/jss . v053.i09.

Peterson, Erik (2017). "The Role of the Information Environment in Partisan Voting". Journal of Politics 79(4), 1191-1204. DOI: 10.1086/692740.

Sewell, Daniel K., Yuguo Chen, William Bernhard, and Tracy Sulkin (2016). "Modelbased longitudinal clustering with varying cluster assignments". Statistica Sinica 26(1), 205-233. DOI: 10.5705/ss.2014.205.

Shiraito, Yuki (2016). "Uncovering Heterogeneous Treatment Effects".

Smidt, Corwin D. (2017). "Polarization and the Decline of the American Floating Voter". American Journal of Political Science 61(2), 365-381. DOI: 10.1111/ajps. 12218.

Stark, Philip B. (2008). "Conservative Statistical Post-Election Audits". Annals of Applied Statistics 2(2), 550-581. DOI: 10.1214/08-A0AS161.

Wedel, Michel and Wagner A. Kamakura (2000). Market Segmentation: Conceptual and Methodological Foundations. 2nd ed. Kluwer Academic Publishers.

Yamauchi, Soichiro (2020). emlogit: A ECM algorithm for the Multinomial Logit Model. URL: https://github.com/soichiroy/emlogit. 


\section{Appendix A: Package Speed Performance}

Figure 6 shows the time required to complete the clustering algorithm on datasets of varying sizes and four alternatives for the number of clusters. I take the entire dataset by Herron and Lewis (2007), which includes data on 3 million voters in 10 Florida counties. I only consider four offices $(J=4)$ and vary the number of voters to analyze.

Time measurements in seconds are plotted on a log-log scale. Both clusterCVR (the method I use in this paper) and the R package poLCA scale relatively well, but poLCA can be an order of magnitude slower in very large datasets.

Because of the collapsing procedure I describe below, the algorithm iterates through unique profiles, instead of individual voters. Without the collapsing, both algorithms will take orders of magnitude longer in large datasets.

Figure 6: Scalability of the Clustering Algorithm

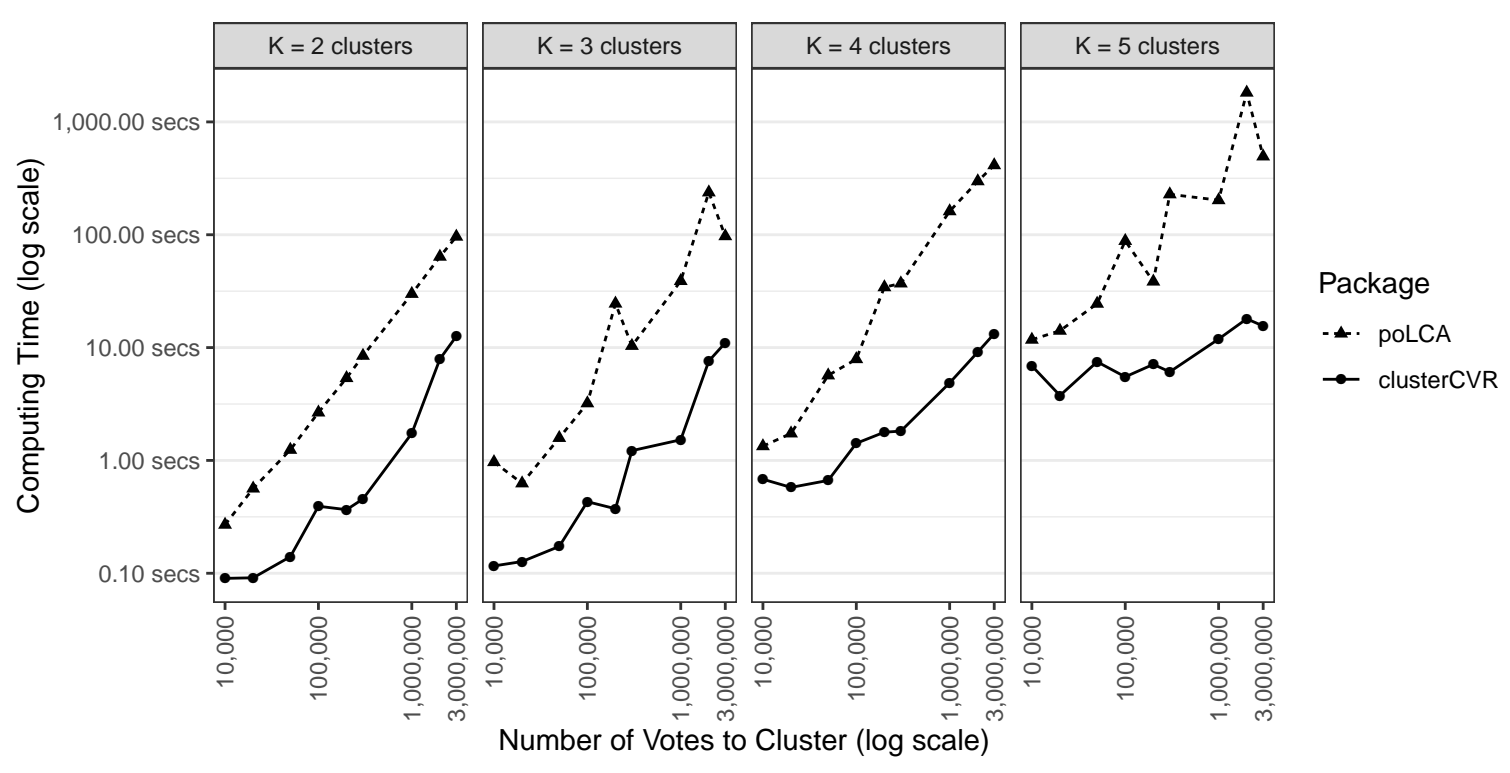




\section{Appendix B: EM Derivations}

This appendix formalizes and derives the key steps of the clustering model and EM algorithm. The notation follows the main text.

\section{B.1 Complete Likelihood}

If we knew the cluster assignment, we would be able to write the complete log-likelihood $\left(\mathcal{L}_{\text {comp }}\right)$. First start with the joint probability of the outcome data and the cluster assignment:

$$
\begin{aligned}
\operatorname{Pr}(\mathbf{Y}, \mathbf{Z} \mid \boldsymbol{\mu}, \boldsymbol{\pi}) & =\operatorname{Pr}(\mathbf{Y} \mid \mathbf{Z}, \boldsymbol{\mu}, \boldsymbol{\pi}) \operatorname{Pr}(\mathbf{Z} \mid \boldsymbol{\pi}) \\
& =\prod_{i=1}^{N} \prod_{j=1}^{J} \operatorname{Pr}\left(Y_{i j} \mid \mathbf{Z}, \boldsymbol{\mu}\right) \prod_{i=1}^{N} \operatorname{Pr}\left(Z_{i} \mid \boldsymbol{\pi}\right) \\
& =\prod_{i=1}^{N} \prod_{j=1}^{J} \prod_{k=1}^{K}\left\{\prod_{\ell=0}^{L} \operatorname{Pr}\left(Y_{i j}=\ell \mid Z_{i}=k\right)^{\mathbf{1}\left(Y_{i j}=\ell\right)}\right\}^{\mathbf{1}\left(Z_{i}=k\right)} \prod_{i=1}^{N} \prod_{k=1}^{K} \operatorname{Pr}\left(Z_{i}=k \mid \boldsymbol{\pi}\right)^{\mathbf{1}\left(Z_{i}=k\right)}
\end{aligned}
$$

Therefore, the complete log-likelihood is:

$$
\begin{gathered}
\mathcal{L}_{\text {comp }}(\boldsymbol{\mu}, \boldsymbol{\pi} \mid \mathbf{Y}, \mathbf{Z})=\sum_{i=1}^{N} \sum_{j=1}^{J} \sum_{k=1}^{K} \sum_{\ell=0}^{L} \mathbf{1}\left\{Y_{i j}=\ell, Z_{i}=k\right\} \log \operatorname{Pr}\left(Y_{i j}=\ell \mid Z_{i}=k, \boldsymbol{\mu}\right) \\
+\sum_{i=1}^{N} \sum_{k=1}^{K} \mathbf{1}\left\{Z_{i}=k\right\} \log \operatorname{Pr}\left(Z_{i}=k \mid \boldsymbol{\pi}\right)
\end{gathered}
$$

To derive the EM algorithm, we first take expectations over the latent variable $Z_{i}$,

$$
\begin{aligned}
\mathbb{E}\left[\mathcal{L}_{\text {comp }}\right]=\sum_{i=1}^{N} & \sum_{j=1}^{J} \sum_{k=1}^{K} \sum_{\ell=0}^{L} \mathbf{1}\left(Y_{i j}=\ell\right) \mathbb{E}\left[\mathbf{1}\left(Z_{i}=k\right)\right] \underbrace{}_{\equiv \log \boldsymbol{\mu}_{k j \ell} \log \operatorname{Pr}\left(Y_{i j}=\ell \mid Z_{i}=k, \boldsymbol{\mu}\right)} \\
& +\sum_{i=1}^{N} \sum_{k=1}^{K} \mathbb{E}\left[\mathbf{1}\left(Z_{i}=k\right)\right] \underbrace{\log \operatorname{Pr}\left(Z_{i}=k \mid \boldsymbol{\pi}\right)}_{=\log \boldsymbol{\pi}_{k}}
\end{aligned}
$$

We represent this unknown quantity as

$$
\zeta_{i k} \equiv \mathbb{E}\left[\mathbf{1}\left(Z_{i}=k\right)\right]
$$


Then the E-step can be the normalized version of the posterior probability marginalized by the mixing proportion,

$$
\widehat{\zeta}_{i k} \propto \pi_{k} \prod_{j=1}^{D} \underbrace{\prod_{\ell=0}^{L}\left(\mu_{k j \ell}\right)^{\mathbf{1}\left(Y_{i j}=\ell\right)}}_{\equiv \boldsymbol{\mu}_{k j, Y_{i j}}}
$$

E-step For each voter $i$, compute the probability that they belong in cluster $k$ :

$$
\zeta_{i k} \leftarrow \frac{\boldsymbol{\pi}_{k} \prod_{j=1}^{J} \boldsymbol{\mu}_{k j, Y_{i j}}}{\sum_{k^{\prime}=1}^{K} \boldsymbol{\pi}_{k^{\prime}} \prod_{j=1}^{J} \boldsymbol{\mu}_{k^{\prime} j, Y_{i j}}}
$$

The M-step is derived by taking the derivatives of $\mathbb{E}\left[\mathcal{L}_{\text {comp }}\right]$ with respect to the model parameters $\boldsymbol{\mu}$ and $\boldsymbol{\pi}$. This leads to a MLE-like M-step:

M-step Take the MLE, as derived in section B.4. For updating $\pi_{k}$, take the simple average of $\widehat{\zeta}_{i k}$ across all $i$. For updating $\widehat{\mu}_{k j \ell}$, take for each $k$ and $\ell$ the sample proportion of the occurrence of $Y_{i j}=\ell$, but weighted by $\widehat{\zeta}_{i k}$ :

$$
\begin{aligned}
& \text { for each } k \text {, update: } \widehat{\pi}_{k} \leftarrow \frac{1}{N} \sum_{i=1}^{N} \widehat{\zeta}_{i k} \\
& \text { for each } k, j, \ell, \text { update: } \quad \widehat{\mu}_{k j \ell} \leftarrow \frac{\sum_{i=1}^{N} \mathbf{1}\left(Y_{i j}=\ell\right) \widehat{\zeta}_{i k}}{\sum_{i=1}^{N} \widehat{\zeta}_{i k}},
\end{aligned}
$$

We iterate through these two steps until convergence.

\section{B.2 Evaluating Convergence}

We evaluate convergence by the observed log likelihood,

$$
\mathbf{L}_{\mathrm{obs}}=\prod_{i=1}^{N} \sum_{k=1}^{K} \pi_{k} \prod_{j=1}^{J} \boldsymbol{\mu}_{k j, Y_{i j}}
$$


So the observed log-likelihood is

$$
\mathcal{L}_{\text {obs }}=\sum_{i=1}^{N} \log \left\{\sum_{k=1}^{K} \pi_{k} \prod_{j=1}^{J} \boldsymbol{\mu}_{k j, Y_{i j}}\right\}=\sum_{i=1}^{N} \log \left\{\sum_{k=1}^{K} \pi_{k} \prod_{j=1}^{J} \prod_{\ell=0}^{L}\left(\mu_{k j \ell}\right)^{\mathbf{1}\left(Y_{i j}=\ell\right)}\right\}
$$

\section{B.3 Speed-Up by Collapsing to Unique Profiles}

Because this EM algorithm deals with discrete data, the algorithm needs only sufficient statistics. In our setting the unique number of voting profiles is much smaller than the number of observations, because vote vectors follow a systematic pattern and most votes are straight-ticket votes. Therefore, we can re-format the dataset so that each row is a unique combination.

Let $u \in\{1, \ldots, U\}$ index the unique voting profiles, and $n_{u}$ be the number of such profiles in the data. We re-cycle the objects $\mathbf{Y}$ and $\boldsymbol{\zeta}$ so that each row indexes profiles rather than voters.

We repeat the EM algorithm described earlier. For each profile $u$, compute the probability that it belong in type $k$ :

$$
\text { for each } u, k \text {, update: } \quad \widehat{\zeta}_{u k} \leftarrow \frac{\boldsymbol{\pi}_{k} \prod_{j=1}^{J} \boldsymbol{\mu}_{k j, Y_{u j}}}{\sum_{k^{\prime}=1}^{K} \boldsymbol{\pi}_{k^{\prime}} \prod_{j=1}^{J} \boldsymbol{\mu}_{k^{\prime} j, Y_{u j}}}
$$

Then given those type probabilities, update with

$$
\begin{aligned}
& \text { for each } k \text {, update: } \widehat{\pi}_{k} \leftarrow \frac{1}{N} \sum_{u=1}^{U} n_{u} \widehat{\zeta}_{u k} \\
& \text { for each } k, j, \ell, \text { update: } \quad \widehat{\mu}_{k j \ell} \leftarrow \frac{\sum_{u=1}^{U} n_{u} \mathbf{1}\left(Y_{u j}=\ell\right) \widehat{\zeta}_{u k}}{\sum_{u=1}^{U} n_{u} \widehat{\zeta}_{u k}}
\end{aligned}
$$

And the observed log-likelihood will also only require looping through the profiles:

$$
\mathcal{L}_{\text {obs }}=\sum_{u=1}^{U} \log n_{u}+\sum_{u=1}^{U} \log \left\{\sum_{k=1}^{K} \pi_{k} \prod_{j=1}^{J} \boldsymbol{\mu}_{k j, Y_{i j}}\right\}
$$




\section{B.4 Derivation of $M$-step}

Recall that the expectation of the likelihood from equation 5 is

$$
\mathbb{E}\left[\mathcal{L}_{\text {comp }}\right]=\sum_{i=1}^{N} \sum_{j=1}^{J} \sum_{k=1}^{K} \sum_{\ell=0}^{L} \mathbf{1}\left(Y_{i j}=\ell\right) \zeta_{i k} \log \mu_{k j \ell}+\sum_{i=1}^{N} \sum_{k=1}^{K} \zeta_{i k} \log \pi_{k}
$$

so to optimize we introduce Langrange multipliers $\lambda$ and $\boldsymbol{\eta}$ for the constraints on $\boldsymbol{\pi}$ and $\boldsymbol{\mu}_{k j}$, respectively:

$$
\widetilde{\mathcal{L}}=\mathbb{E}\left[\mathcal{L}_{\text {comp }}\right]-\lambda\left(\sum_{k=1}^{K} \pi_{k}-1\right)-\sum_{k=1}^{K} \sum_{j=1}^{J} \eta_{k j}\left(\sum_{\ell=0}^{L} \mu_{k j \ell}-1\right)
$$

Then, for $\boldsymbol{\pi}$ we have that

$$
\frac{\partial}{\partial \pi_{k}} \widetilde{\mathcal{L}}=\frac{\sum_{i=1}^{N} \zeta_{i k}}{\pi_{k}}-\lambda=0
$$

along with the constraint $\sum_{k=1}^{K} \pi_{k}=1$. Notice that when we sum the FOC for $\boldsymbol{\pi}$ across $k$, the first condition becomes $\sum_{k=1}^{K} \pi_{k}=\frac{1}{\lambda} \sum_{k=1}^{K} \sum_{i=i}^{N} \zeta_{i k}$, and because the LHS sums to 1 due to the constraint and in the RHS $\sum_{i=1}^{N} \sum_{k^{\prime}=1}^{K} \zeta_{i k^{\prime}}$ sums to $N$, we have $\lambda=N$.

Separately, for $\boldsymbol{\mu}_{k j}$ we have that

$$
\frac{\partial}{\partial \mu_{k j \ell}} \widetilde{\mathcal{L}}=\frac{\sum_{i=1}^{N} \mathbf{1}\left(Y_{i j}=\ell\right) \zeta_{i k}}{\boldsymbol{\mu}_{k j \ell}}-\eta_{k j}=0
$$

along with constraint $\sum_{\ell=0}^{L} \mu_{k j \ell}=1$. Once we sum the FOC for $\boldsymbol{\mu}$ across $\ell$ the first condition becomes $\sum_{\ell=0}^{L} \mu_{k j \ell}=\frac{1}{\eta_{k j}} \sum_{i=1} \sum_{\ell=0} \mathbf{1}\left(Y_{i j}=\ell\right) \zeta_{i k}$, and because the LHS again sums to 1 and in the RHS $\sum_{i=1}^{N} \sum_{\ell=0}^{L} \mathbf{1}\left(Y_{i j}=\ell\right) \zeta_{i k}$ sums to the prevalence of the weights $\sum_{i=1}^{N} \zeta_{i k}$, we get $\eta_{k j}=\sum_{i=1}^{N} \zeta_{i k}$.

Together, the above imply that

$$
\pi_{k}=\frac{1}{N} \sum_{i=1}^{N} \zeta_{i k} \quad \text { and } \quad \mu_{k j \ell}=\frac{\sum_{i=1}^{N} \mathbf{1}\left\{Y_{i j}=\ell\right\} \zeta_{i k}}{\sum_{i=1}^{N} \zeta_{i k}}
$$

